https://doi.org/10.23913/ride.v10i20.652

Artículos Científicos

\title{
Etapas de ejecución del programa de mentoría de la Unidad Académica de Medicina Veterinaria y Zootecnia de la Universidad Autónoma de Zacatecas
}

Stages of Execution of the Mentoring Program of the Unidad Académica de Medicina Veterinaria y Zootecnia of the Universidad Autónoma de Zacatecas

Etapas da execução do programa de mentoria da Unidade Acadêmica de Medicina Veterinária e Pecuária da Universidade Autônoma de Zacatecas

Fabiola Lydie Rochin Berumen Universidad Autónoma de Zacatecas, Unidad Académica de Medicina Veterinaria y Zootecnia, México fabiolauaz@outlook.com https://orcid.org/0000-0002-8676-7768

Francisco Javier Gutiérrez Piña Universidad Autónoma de Zacatecas, Unidad Académica de Medicina Veterinaria y Zootecnia, México

fj_gp@yahoo.com.mx https://orcid.org/0000-0001-5743-254X

Daniel Rodríguez Tenorio Universidad Autónoma de Zacatecas, Unidad Académica de Medicina Veterinaria y Zootecnia, México rtenorio00@hotmail.com https://orcid.org/0000-0002-2113-5911

Juan Antonio Ramírez Chequer Universidad Autónoma de Zacatecas, Unidad Académica de Medicina Veterinaria y Zootecnia, México cheque-r@hotmail.com https://orcid.org/0000-0001-6400-0390 


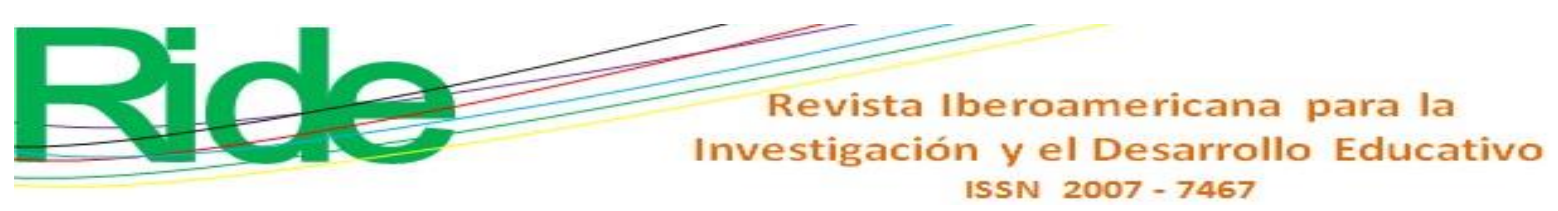

a estudiantes de recién ingreso en la Unidad Académica de Medicina Veterinaria y Zootecnia de la UAZ (Aguilar y Manzano, 2018).

\section{Antecedentes de la mentoría}

La educación ha formado parte de la vida del ser humano. Sin embargo, "aún existen dilemas que hoy en día enfrenta la sociedad para sostener y garantizar el bienestar de sus poblaciones" (Hernández, 2016, p. 18). De acuerdo con la Declaración Incheon Educación 2030 (citada en Hernández, 2016), es necesario trabajar en la capacidad de propuestas y respuestas de los sistemas educativos, principalmente en la universidad; sobre la forma de utilizar y emplear recursos, formar e informar al profesorado, así como de ajustar las estructuras curriculares existentes en beneficio de sus estudiantes. Para la comprensión de la mentoría e identificar más adelante sus etapas, en este apartado se expondrán las bases teóricas de esta investigación: desde antecedentes hasta definiciones, tipos y programas de mentoría.

La mentoría ha presentados varias facetas a lo largo de la historia. Y según la revisión literaria, hay varios modelos, sistemas y programas de mentoría implementados especialmente en universidades, las cuales han sido espacios referentes para el apoyo a estudiantes. Díaz y Bastías (2013) establecen que el concepto de mentoría proviene de la mitología griega. En la Odisea, Homero narra la historia de Ulises, rey de Ítaca, quien se dirigió a su leal amigo Mentor (disfrazado de la diosa Atena) para pedirle que velara por su hijo mientras él iba a luchar en la guerra de Troya. Para los griegos, un mentor era considerado un padre adoptivo, una persona responsable del desarrollo físico, social, intelectual y espiritual de los jóvenes (Díaz y Bastías, 2013).

Las universidades europeas acogieron las bondades de la mentoría como mecanismo de apoyo en la educación de las personas. En la Universidad de Oxford, por ejemplo, emergieron los llamados dons, mentores cuyo compromiso los llevó al grado de radicarse en las instalaciones de la universidad para vivir de cerca la realidad estudiantil y, a la vez, instruir en aspectos relevantes para la adaptación del alumno.

La mentoría estuvo activa también un par de siglos atrás, en el siglo XVI. Cuando iniciaron las agrupaciones comerciales, artesanales y profesionales, se sintió la necesidad de mano de obra calificada para la renovación industrial; personal con experiencia y que estuviera enfocado en prestar atención en el trabajador novel para impulsarlo en el desarrollo de las aptitudes y habilidad requeridas. Los oficios vieron florecer así la relación maestro-aprendiz (Aguilar y Manzano, 2018). 


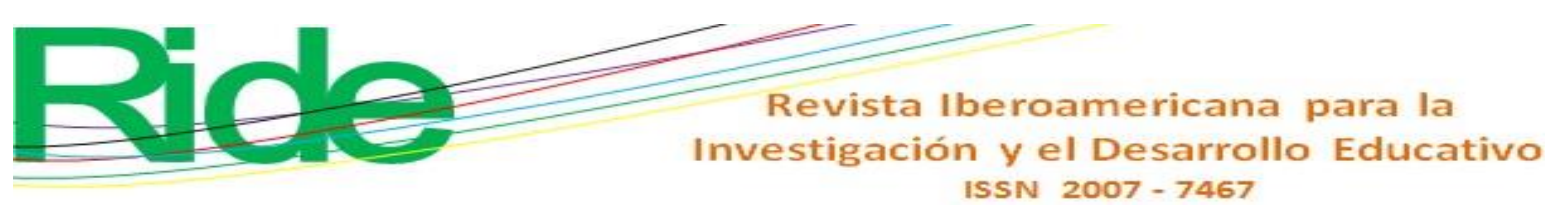

De esta forma, la mentoría delineada durante la Revolución Industrial, que inició en la segunda mitad del siglo XVIII (1750-1780) en Inglaterra, se extendió décadas después a Europa occidental y Norteamérica. Llegado a este punto no está de más recordar que "los cambios tecnológicos introducidos por la Revolución Industrial supusieron una ruptura con el pasado mucho más drástica que cualquier otra invención desde la rueda" (Chaves, 2004).

El desarrollo de la mentoría incluye intervenciones de diversas instituciones educativas de nivel superior. Tal es el caso de la Red Temática de Mentoría, la cual pretende ser un punto de encuentro en el que profesores y alumnos mentores de cualquier universidad española, así como profesionales, puedan compartir experiencias, objetivo, metodologías y recursos, entre otros (Sánchez, 2012). En dicha red se involucran las siguientes universidades:

Universidad Politécnica de Madrid (coordinación),

Universidad Complutense de Madrid,

Universidad Europea de Madrid,

Universidad de Las Palmas de Gran Canarias,

Universidad de Oviedo,

Universidad Carlos III de Madrid,

Universidad Politécnica de Cartagena,

Universidad Nacional de Educación a Distancia (UNED),

Universidad de Alcalá de Henares,

Universidad Politécnica de Valencia,

Universidad de Granada,

Universidad de Cádiz,

Universidad de Málaga y

Universidad de Castilla La Mancha (Sánchez, 2012).

Aunado a ello, y de forma particular, la UNED, en colaboración con el grupo de investigación Desarrollo y Orientación de la Carrera Profesional (Docap), ha llevado a cabo una experiencia piloto poniendo en marcha un modelo específico de mentoría orientada a estudiantes de nuevo ingreso (Manzano, Martín, Sánchez, Rísquez y Suárez, 2012). Dicho modelo contempla a dos figuras fundamentales: "la del consejero/a, que es desempeñada por un/a profesor/a tutor/a, y la del compañero/a-mentor/a" (Manzano et al., 2012). 


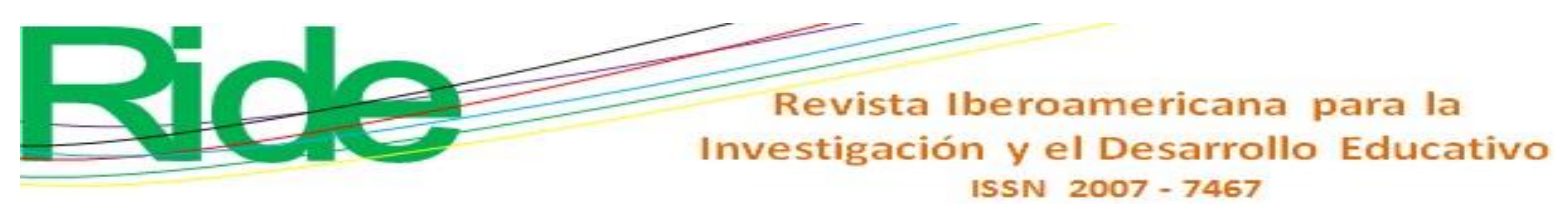

De igual manera, diversas universidades plantean programas de orientación estudiantil, sin necesidad de ser parte de una red de mentoría, con el fin de poner en marcha mecanismos de asistencia, orientación y formación dirigidos a estudiantes de nuevo ingreso, donde son los propios alumnos de niveles avanzados quienes asumen el rol de mentor, gracias a lo cual, además, se potencian capacidades sociales, entre ellas la de liderazgo, así como aspectos académicos, principalmente estrategias metodológicas para interpelar de mejor manera al aprendiz, es decir, vislumbran la mentoría dentro de la práctica pedagógica como un ejercicio fundamental no solo en la formación de sus estudiantes, sino en la de sus profesores. Dicho de otra manera, "la mentoría es la instancia de vínculo entre la universidad y los establecimientos educacionales" (Díaz y Bastías, 2013, p. 301).

La mentoría entre iguales es una estrategia de orientación. Se trata de alumnos de nivel superior con experiencia ayudando a estudiantes de recién ingreso para facilitarles la pronta adaptación en el nuevo escenario educativo, con la supervisión de un docente tutor, quien orienta, guía y acompaña al mentor para el logro de objetivos, según una planeación anteriormente determinada (Romero, 2014).

La mentoría se asocia, por un lado, con una relación vertical, esto es, cuando el mentor es una persona de mayor estatus y el mentorado se encuentra en una posición menor, sea por su edad o nivel de conocimientos. Por el otro, también se puede presentar de manera horizontal, y esto ocurre cuando el apoyo es brindado por un par que cursa el mismo nivel educativo, aunque con u poco más de experiencia, es decir, un compañero experimentado (Manzano et al., 2012).

La mentoría académica es entendida como un proceso de acompañamiento de carácter formativo, orientador e integral que tiene como finalidad facilitar a los estudiantes herramientas y ayuda necesaria para conseguir con éxito todos los objetivos académicos, personales y profesionales que les plantea su institución (Mayoral, Pontes, Melo y Errandosoro, 2012). Para este mismo grupo de autores, la mentoría, entendida como un mecanismo de acompañamiento, busca la mejora del rendimiento escolar y la integración social de los alumnos a través del acceso a una educación de calidad para todos, en la que se requiere enriquecer el entorno educativo e implicar a la comunidad local (Mayoral et al., 2012).

La mentoría grupal, según Gómez y Eisman (2011), previene, identifica y favorece problemas de orden escolar, personal, familiar o social que afectan su proceso formativo y canaliza a instancias internas para su atención; la mentoría individual atiende asuntos que influyen en el proceso educativo, mejora las condiciones de aprendizaje, desarrolla valores, actitudes, hábitos y 


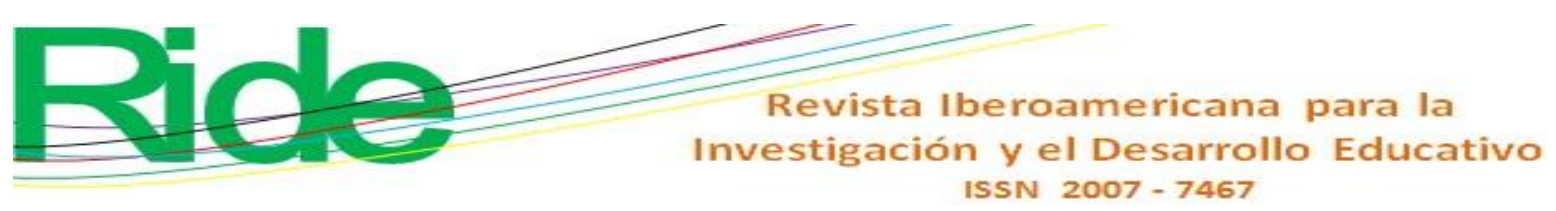

habilidades que contribuyen a la integridad de la formación profesional y humana, se planifica por sesiones y se registra la evolución.

La tutoría entre pares es una manera más de llevar a cabo esta práctica, como ya se ha visto. En ella, los mismos estudiantes, previamente capacitados, brindan asesoría y apoyo a sus compañeros para solucionar un problema, completar una tarea, aprender una estrategia o dominar un procedimiento dentro de un programa previamente planificado. Parece ser que este es el concepto con el que se empata la estrategia de relación mentora: persigue como objetivo la formación integral de los estudiantes, atender al estudiante a partir de sus necesidades específicas (Casado, Lezcano y Colomer, 2015).

En suma, esta práctica la ejecutan alumnos identificados y entrenados por sus cualidades personales y académicas para apoyar a otros estudiantes. Son individuales o grupales y tienen el objetivo de solucionar un problema, completar una tarea, aprender alguna estrategia, dominar un procedimiento. Asimismo, apoya la función de los profesores tutores que aprovechan las capacidades de alumnos sobresalientes de los últimos semestres. Aunque se parte más de aspectos académicos, se dice que quien dé asistencia a otros, lo haga solo porque está adelantado en sus estudios (Quevedo y Medeiros, 2010).

Karina Fuerte (23 de marzo de 2018) expone que la innovación del programa Redes de Tutoría, un proyecto que nació hace más de veinte años en México, consiste en un cambio fundamental en el sistema pedagógico: ahora no es el maestro la única fuente de conocimiento en el aula, pues cada uno de los alumnos tiene la capacidad de aprender y de enseñar a sus pares. Además, representa una práctica democrática, donde todos tienen algo valioso que compartir. Planteamiento que también se refleja en el artículo de López, Lozano y Xique (2019), en donde se hace mención de lo siguiente:

La labor del mentor es idónea para acompañar a los alumnos en su etapa de formación creando ambientes de confianza, aprendizaje, reflexión y conocimiento. Actualmente, el docente debe buscar nuevas estrategias de enseñanza-aprendizaje para coadyuvar en la formación de recursos humanos competentes en los nuevos escenarios de la educación terciaria; por ende, es importante la participación de los mismos estudiantes para forjar a sus pares y sean ellos mismos quienes sean sus propios mentores (p. 2).

La mentoría puede ser una estrategia mediante la cual desaparezcan las barreras sociales, culturales y económicas. También para acabar con estereotipos como el de que solo el maestro enseña dentro 


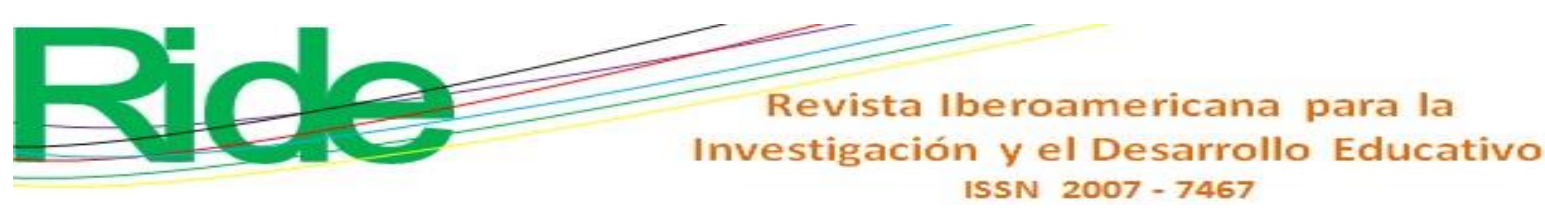

del aula, y así atender a los educandos mediante enfoques pedagógicos dinámicos y colaborativos (Hernández, 2016).

Volviendo a la Declaración Incheon Educación 2030 (Organización de las Naciones Unidas para la Educación, la Ciencia y la Cultura [Unesco], 2016), en particular al punto 50, el cual gira en torno a la educación para todos y con miras al futuro, ahí se menciona la necesidad de crear programas con servicios de orientación y asesoría adecuada, lo que hace pensar en la necesidad de una alternativa de atención distinta que sea más orientativa como puede ser la mentoría.

Teniendo en cuenta todo lo anterior, la presente propuesta para la implementación de mentoría dirigida a estudiantes de recién ingreso es definir las etapas de ejecución de la mentoría de la Unidad Académica de Medicina Veterinaria y Zootecnia de la UAZ, las cuales tienen una secuencia sistemática desde su inicio hasta el cierre del programa implementado, independientemente de si es mentoría grupal o individual.

\section{Materiales y métodos \\ Primera etapa: conformar el equipo mentor}

Designar un coordinador especialista en educación. Esta función puede cumplirla el docente.

Reclutar a estudiantes voluntarios que deseen cumplir el rol de mentores con actitud de servicio.

Selección definitiva de mentores por rendimiento académico de tercer semestre a octavo semestre.

\section{Segunda etapa: capacitación de mentores}

Una capacitación dirigida a los mentores, la cual es brindada por el coordinador de mentorías de la Unidad Académica de Medicina Veterinaria y Zootecnia de la UAZ, para que exista una interacción dinámica, donde el estudiante con problemas de aprendizaje es orientado por un estudiante sobresaliente capacitado.

Capacitar al equipo mentor con reuniones periódicas.

Aplicar la primera sesión de trabajo mediante un taller de inducción para la familiarización de estudiantes de recién ingreso y mentores; para conocer sobre los ambientes físicos y virtuales del nuevo contexto educativo; para el conocimiento de las estrategias metodológicas que aplican los docentes, y para la presentación de los programas académicos.

Desarrollo de reuniones periódicas entre mentor y mentorados, cumpliendo de manera sistemática el cronograma planteado en el programa de mentoría. 


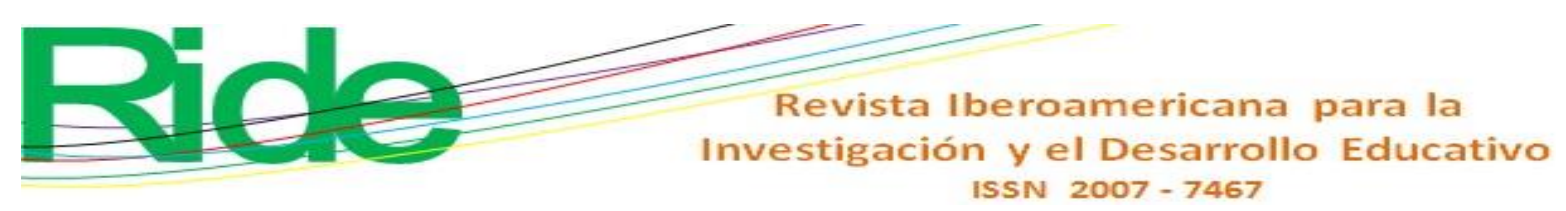

\section{Tercera etapa: asignación de materias}

Al mentor se le asigna las materias de primer y segundo semestre que están establecidas en el plan de estudios de la Lic. de Veterinaria y Zootecnia, por medio del coordinador de mentorías de la Unidad Académica de Medicina Veterinaria y Zootecnia, tomando en cuenta un diálogo previo con el alumno mentor para identificar qué materias resultan más fáciles de asesorar para ayudar a los alumnos de nuevo ingreso.

\section{Cuarta etapa: diagnóstico de estudiantes de recién ingreso}

Reclutar estudiantes de recién ingreso (particularmente primer y segundo semestre).

Aplicar test a estudiantes de recién ingreso para determinar sus necesidades de atención.

\section{Quinta etapa: asignación de mentorados}

Definir las necesidades de atención de los estudiantes: académica, vocacional, personal, social, económica, preferencias, situación del hogar, administrativa; con base en ello el coordinador asignará los mentorados que necesiten alguna asesoría académica en alguna materia en específico.

\section{Sexta etapa: diseño de formatos}

Selección de estrategias metodológicas, técnicas e instrumentos a aplicar por el coordinador de mentorías y mentor en el desarrollo del programa de mentoría, considerando los resultados del diagnóstico y aplicando los formatos de registro de estudiante mentorado, registro de sesiones mentor-mentorado, registro de estudiante mentor (ver anexos).

Los formatos fueron elaborados por el Centro Académico de Servicios Estudiantiles (CASE) de la UAZ.

\section{Séptima etapa: implementación y seguimiento}

Verificar el cumplimiento de actividades y fechas programadas establecidas durante el semestre en activo.

Redactar un informe que evidencia el seguimiento al cumplimiento de acciones planeadas. 


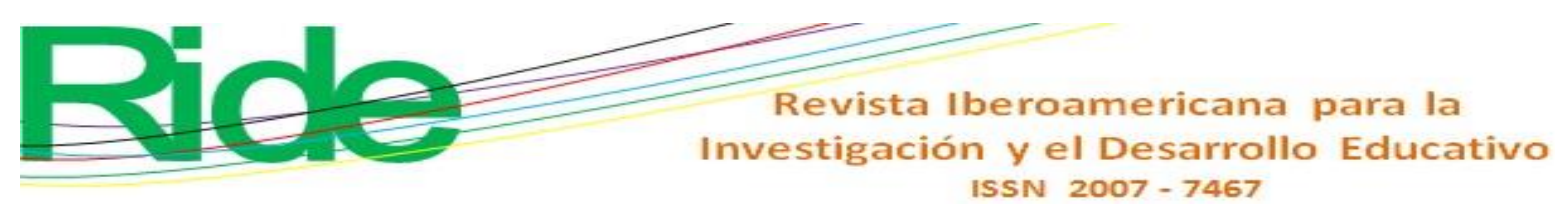

Octava etapa: evaluación del programa de mentoría

Análisis de resultados.

Redacción del informe final de evaluación del programa de mentoría.

\section{Discusión}

El programa de mentoría inició en el año 2010 en el CASE de la UAZ y se incorporó de manera formal al Programa Institucional de Mentorías (Cardoso, 25 de octubre 2010) para dar apoyo a estudiantes de nuevo ingreso de las diversas unidades académicas de dicha universidad, regulado por la Coordinación de Mentorías perteneciente al CASE.

La Unidad Académica de Medicina Veterinaria y Zootecnia de la UAZ se incorpora a este programa en el año 2016. Como enlace con el CASE se designa a la Dra. Fabiola Lydie Rochin Berumen en el periodo 2016-2020, docente adscrita a la unidad académica en cuestión, siguiendo las estrategias de apoyo y orientación para los alumnos de nuevo ingreso para su mejora académica, evaluando los resultados de los alumnos (mentorados) en lo concerniente al promedio final de la materia en la cual se le brindó mentoría; la información se ejecuta en una base de datos, la cual se remite al director de la unidad y a la coordinación del CASE para mostrar los resultados obtenidos. El objeto de este estudio pretendió dar a conocer las etapas de ejecución del Programa Institucional de Mentorías a la comunidad universitaria, debido a que los participantes son los docentes y estudiantes, quienes colaboran en un programa de formación para conformar un equipo mentor con su pertinente capacitación. A partir del análisis cualitativo se puede observar un seguimiento e innovación en el programa de mentoría, labor que realizan los estudiantes sobresalientes de la Unidad Académica de Medicina Veterinaria y Zootecnia. Esta percepción está en concordancia con el programa de mentoría que se imparte en la Universidad de La Laguna, con el objetivo de orientar e impulsar en los estudiantes la vocación de la enseñanza en diferentes áreas de conocimiento, dada la oportunidad de realizar actividades formativas, además de adquirir competencias y experiencia en la transmisión de sus conocimientos (Borges, Rodríguez, Aguirre, Dorta y Noda, 2018). 


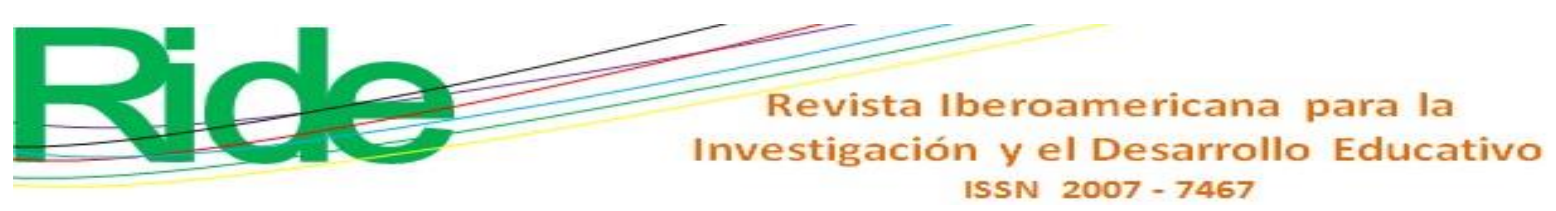

\section{Conclusiones}

El Programa Institucional de Mentorías de la UAZ se lleva a cabo con el propósito de lograr una pronta adaptación en estudiantes de nuevo ingreso y lograr con ello disminuir la inseguridad en los estudiantes al enfrentarse a nuevos escenarios educativos, nuevos docentes, nuevas asignaturas, nuevas estrategias y nuevos compañeros; para una pronta adaptación en el nuevo escenario educativo, evitar la deserción escolar y mejorar los resultados académicos.

En este sentido, la tutoría es una estrategia valiosa para proporcionar a los estudiantes de elementos emocionales e instrumentos que les permitan alcanzar sus objetivos desde sus primeros pasos en los espacios universitarios, mientras que, a los estudiantes de apoyo, quienes necesitan alcanzar la meta de un título universitario, se les otorgan habilidades que les servirán en su ejercicio profesional o continuación académica.

Sin duda la prevalencia y el impacto positivo de la tutoría ha generado una gran cantidad de investigación en ciencias sociales; con ello se busca garantizar que se produzcan los resultados más positivos para los estudiantes dentro de cualquier programa de tutoría (Coles, 2011).

En este estudio se encontraron limitaciones a raíz del poco conocimiento del proceso de ejecución y la poca información del programa de mentorías. Es por eso por lo que, para fomentar el diseño y la puesta en marcha del Programa Institucional de Mentorías, en esta investigación se concluye con una propuesta de ocho etapas de ejecución dentro de la Unidad Académica de Medicina Veterinaria y Zootecnia de la UAZ; etapas que permitirán generar una adecuada organización, construcción y ejecución del programa y sus participantes. Para ello, además, es primordial la tarea de la Unidad Académica de Medicina Veterinaria y Zootecnia de garantizar la evaluación y seguimiento de los resultados con artículos, como en este caso, que propicien una mejor integración de elementos innovadores que salvaguarden la intención principal del programa: reducir los casos de alumnos que desertan sus estudios. 


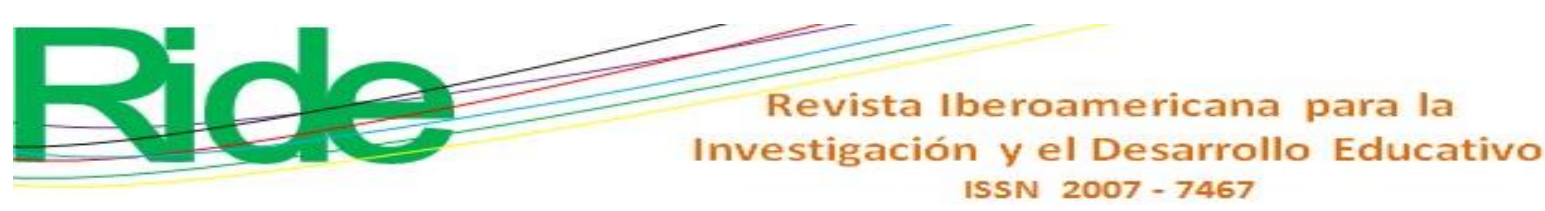

\section{Referencias}

Aguilar, N. L. y Manzano, N. (2018). La mentoría en el nivel universitario: etapas para su implementación. Revista Universidad y Sociedad, 10(3), 255-262.

Borges, Á., Rodríguez, M., Aguirre, T., Dorta, M. J. y Noda, F. J. (2018). Evaluación de la Primera Edición del Programa de Mentorías Comparte con la Universidad de La Laguna. Revista Talento, Inteligencia y Creatividad, 5(9), 16-32. Recuperado de http://www.talincrea.cucs.udg.mx/sites/default/files/Evaluacio\%CC\%81n $\% 20 \mathrm{de} \% 201 \mathrm{a} \% 2$ 0Primera\%20Edicio\%CC\%81n\%20del\%20Programa\%20de\%20Mentori\%CC\%81as\%20 Comparte\%20con\%201a\%20Universidad\%20de\%20La\%20Laguna\%20(ULL).pdf.

Cardoso, M. de J. (25 de octubre de 2010). Programa Institucional de Mentorías. Programa Institucional de Mentorías CASE. Recuperado de http://mentoriacaseuaz.blogspot.com/.

Casado, R., Lezcano, F. y Colomer, J. (2015). Diez pasos clave en el desarrollo de un programa de mentoría universitaria para estudiantes de nuevo ingreso. Revista Electrónica Educare, 19(2), 155-179. Recuperado de https://doi.org/10.15359/ree.19-2.10.

Chaves, J. (2004). Desarrollo tecnológico en la Primera Revolución Industrial. Norba. Revista de Historia, 17, 93-109.

Coles, A. (2011). The role on mentoring in college access and success. Research to Practice Brief, $12(1), 43$.

Díaz, C. y Bastías, C. (2013). Los procesos de mentoría en la formación inicial docente. Revista Internacional de Investigación en Ciencias Sociales, 9(2), 301-315.

Fuerte, K. (13 de marzo de 2018). El diálogo como herramienta de aprendizaje: Redes de Tutoría. Observatorio de Innovación Educativa. Recuperado de https://observatorio.tec.mx/edunews/el-dialogo-como-herramienta-de-aprendizaje-redes-de-tutoria.

Gómez, J. A. y Eisman, E. M. (2011). La mentoría como elemento de mejora en el proceso de enseñanza - aprendizaje. Ponencia presentada en las XVII Jornadas de Enseñanza Universitaria de la Informática. Sevilla, del 5 al 8 de julio de 2011. Recuperado de http://www.sevillacb.com/es/agenda/ver/78?slug=jornadas-de-ensenanza-universitaria-dela-informatica-jenui.

Hernández, N. (2016). Reflexión teórica sobre la Declaración de Incheon Educación 2030 "Hacia una educación inclusiva y equitativa de calidad y un aprendizaje a lo largo de la vida de todos”. Revista Nacional e Internacional de Educación Inclusiva, 9(2), 18-36. 


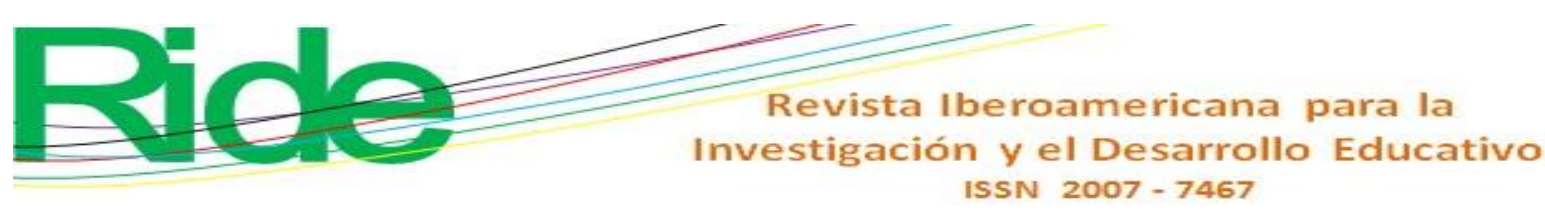

López, M. L., Lozano, I. y Xique, M. A. (2019). La mentoría en la Educación Superior. El caso de la Facultad de Lenguas de la Benemérita Universidad Autónoma de Puebla (BUAP). Revista Dilemas Contemporáneos: Educación, Política y Valores, 7(1), 1-15.

Manzano, N., Martín, A. Sánchez, M., Rísquez, A. y Suárez, M. (2012). El rol del mentor en un proceso de mentoría universitaria. Educación XX1, 15(2), 93-118. Recuperado de http://www.redalyc.org/articulo.oa?id=70624504002.

Mayoral, L., Pontes, H., Melo, P. T. y Errandosoro, F. (2012). Red de mentoría emprendedora en el sector de las Tecnologías de la Información y la Comunicación: La diversidad de los roles sociales y la fuerza de los lazos débiles como soportes de la innovación tecnológica en Tandil, Argentina. Cuadernos de Administración, 25(45), 137-162.

Oliveros, L., García, M., Ruiz de Miguel, C. y Valverde, A. (2004). Innovación en la Orientación Universitaria. Una experiencia: Red de estudiantes mentores en la Universidad Complutense (REMUC). Contextos Educativos. Revista de Educación, 6-7(2003-2004), 331-354. Recuperado de https://doi.org/10.18172/con.543.

Organización de las Naciones Unidas para la Educación, la Ciencia y la Cultura [Unesco]. (2016). Declaración de Incheon y Marco de Acción para la realización del Objetivo de Desarrollo Sostenible 4. Organización de las Naciones Unidas para la Educación, la Ciencia y la Cultura.

Quevedo, T. y de Medeiros, A. M. (2010). El trabajo del mentor: Análisis de los feedbacks de diarios reflexivos a lo largo de un proceso de mentoría en grupo. Revista Iberoamericana de Educación, 52(6), 1-13.

Romero, G. (2014). ¿Coaching? Debates IESA, 19(1), 12-15. 


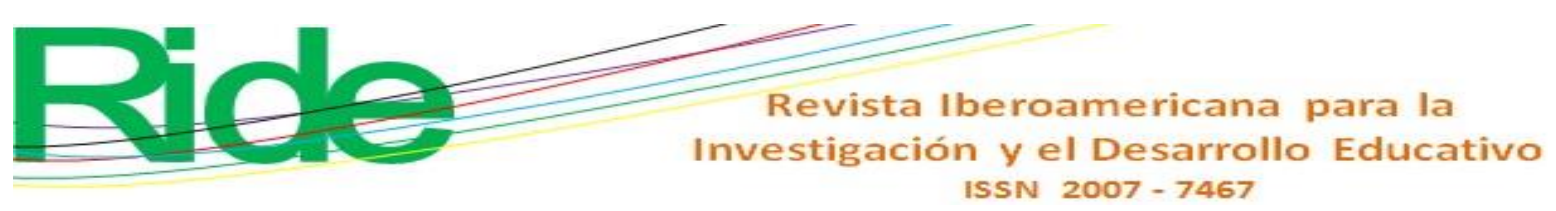

\section{Anexos}

Figura 1. Documento para el registro de sesiones mentor-mentorado de la Unidad Académica de Medicina Veterinaria y Zootecnia de la UAZ

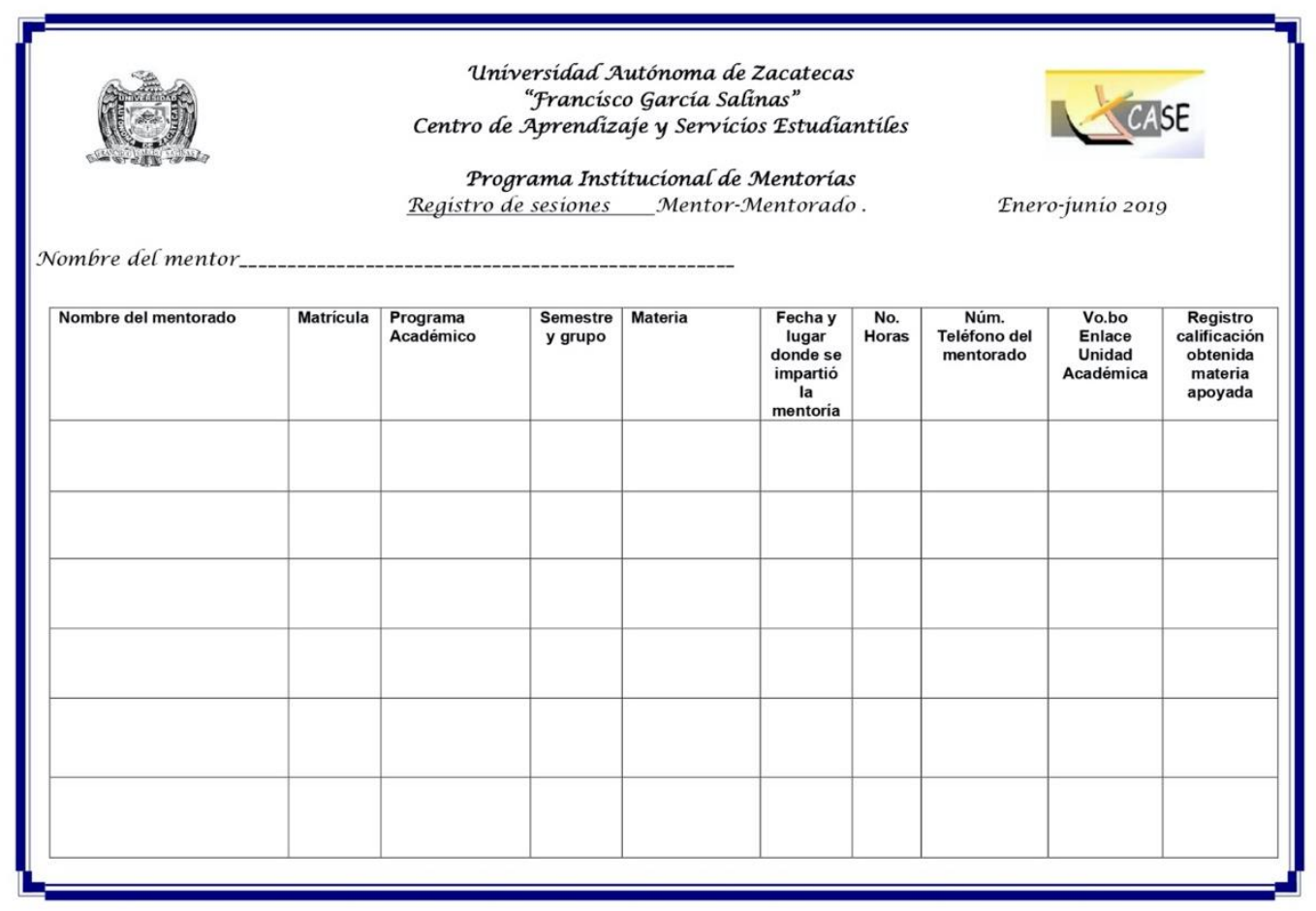

Fuente: CASE de la UAZ 


\section{Revista Iberoamericana para la Investigación y el Desarrollo Educativo \\ ISSN 2007 - 7467}

Figura 2. Documento para el registro del estudiante mentorado de la Unidad Académica de

Medicina Veterinaria y Zootecnia de la UAZ

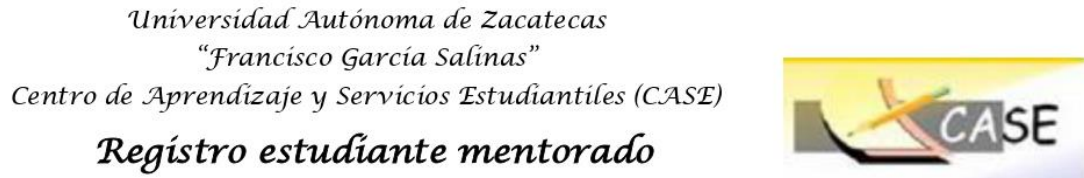

Matrícula

Semestre:

Grupo:

Fecha de nacimiento:

Institución en la que cursó el bachillerato:

Nombre:

Unidad Académica:

Programa Académico:

Teléfono celular:

Correo electrónico:

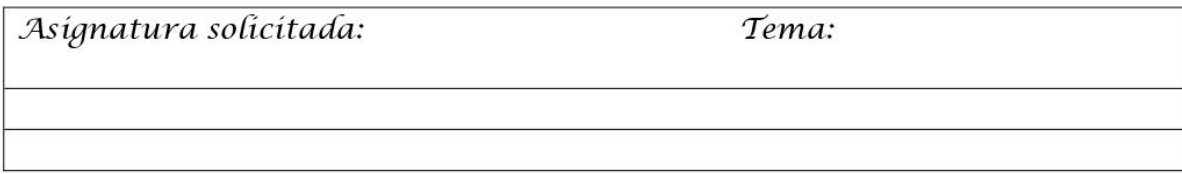

\begin{tabular}{|l|l|l|l|l|l|l|}
\hline $\begin{array}{l}\text { Dias y horarios } \\
\text { disponibles: }\end{array}$ & Lunes & Martes & Miércoles & Jueves & Viernes & otros \\
\hline & & & & & & \\
\hline
\end{tabular}

Reporte de las calificaciones de los exámenes parciales de la materia (s) solicitada (s):

Primer_______segundo_______tercero ___________cuarto

Primer_______segundo_______tercero ___________cuarto______Calif. Final____-_

Nombre del mentor asignado:

Programa académico mentor asignado:

Fecha de registro

Fuente: CASE de la UAZ 


\section{Revista Iberoamericana para la Investigación y el Desarrollo Educativo ISSN 2007 - 7467}

Figura 3. Documento para el registro del estudiante mentor de la Unidad Académica de Medicina Veterinaria y Zootecnia de la UAZ
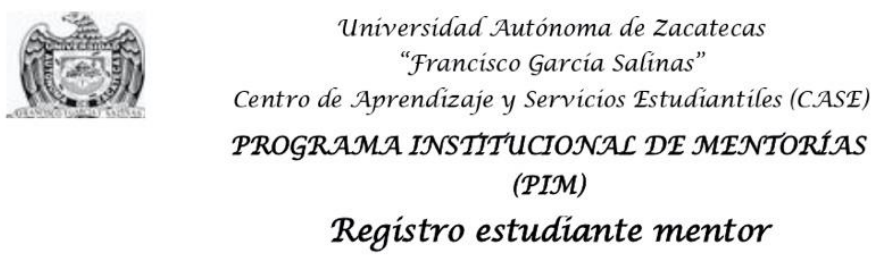

Nombre del mentor: Correo electrónico:

Asignatura a asesorar:

\begin{tabular}{|c|c|c|c|c|c|c|}
\hline \multicolumn{2}{|c|}{} & & & & & \\
\hline Días disponibles: & Lunes & Martes & Miércoles & Jueves & Viernes & otros \\
\hline & & & & & & \\
Horarios disponibles: & & & & & & \\
\hline
\end{tabular}

$\mathcal{N}$ ota:: *El lugar donde se realizará la mentoría será únicamente en instalaciones de la UAZ.

*Al inicio de cada semestre deberá renovar su inscripción como mentor.

Fuente: CASE de la UAZ 